\title{
Strain Differences in Eating and Drinking Evoked by Electrical Stimulation of the Hypothalamus
}

\author{
GUY MITTLEMAN AND ELLIOT S. VALENSTEIN' \\ Department of Psychology and Neuroscience Laboratory, University of Michigan, Ann Arbor, MI 48109
}

Received 27 October 1980

\begin{abstract}
MITTLEMAN, G. AND E. S. VALENSTEIN. Strain differences in eating and drinking evoked by electrical stimulation of the hypothalamus. PHYSIOL. BEHAV. 26(3) 371-378, 1981.-Electrical stimulation of the lateral hypothalamus (ESLH) has been shown to produce individual response differences that cannot be attributed to the neuroanatomical locus of the electrode. The purpose of the present experiment was to investigate strain differences in the incidence of eating and drinking evoked by ESLH. The responses of 49 Long-Evans and 51 Sprague-Dawley male rats implanted with bilateral hypothalamic electrodes were studied. Animals from these two strains do not differ in their normal food or water consumption. Analysis of the responses to ESLH demonstrated that a significantly greater number of Long-Evans rats ate food and/or drank water during ESLH than did the Sprague-Dawley rats. These results could not be attributed to differences in electrode placements, or rearing conditions. In addition to strain differences, the importance of individual differences within each strain was demonstrated by the fact that both electrodes in a given animal commonly evoked the same behavior. Hypotheses to explain these results are discussed.
\end{abstract}

Electrical stimulation Lateral hypothalamus Strain differences Long-Evans Sprague-Dawley Evoked eating and drinking Stimulus-bound behavior

IN response to electrical stimulation of the lateral hypothalamus, rats as well as all other mammals tested display a variety of different behaviors including eating, drinking, gnawing, hoarding, grooming, aggression, retrieval of young and male copulatory behavior (see [60] for a review). Individual animals usually exhibit only a few of the total list of behaviors that have been evoked by hypothalamic stimulation and a great many animals only display an increase in locomotor activity. Although gross anatomical differences in electrode placement and aspects of the testing conditions probably account for some of this behavioral variability, it has been demonstrated that even with indistinguishable electrode placements and experimental procedures, animals show very different responses to brain stimulation $[22,63]$. Moreover, rats with bilateral hypothalamic electrodes implanted at different hypothalamic sites show a strong tendency to display the same behavior from both electrodes [63]. These results indicate that the behavioral response to brain stimulation cannot be adequately predicted from the anatomical locus of the electrodes and provide the justification for postulating that individual animals have a "prepotent" tendency to respond in a characteristic manner [58].

The results of other studies have also emphasized the importance of individual response tendencies. Karli et al. [30] and Panksepp [43], for example, observed that the natural mouse-killing behavior of rats was a good predictor of the effectiveness of hypothalamic stimulation to elicit mouse killing. Panksepp concluded that, "...the electrically elicited response was probably not determined by specific functions of the tissue under the electrode but by the personality of the rat." Using another approach, Wise [67] tested rats for evoked eating and drinking using moveable hypothalamic electrodes. Animals that displayed an evoked consummatory response both ate and drank, and continued to do so as electrodes were advanced as much as $1.5 \mathrm{~mm}$ in a dorsal to ventral direction through the hypothalamus. Furthermore, within the limits of the positive area, movement of the electrode had little effect on current threshold for evoking eating and drinking. In sharp contrast, negative animals failed to eat or drink at any stimulated site. Wise concluded that the variability between animals reflects individual response tendencies rather than differences in the site of stimulation.

More recently, Bachus and Valenstein [3] used DC electric current to destroy hypothalamic cells and fibers surrounding stimulating electrodes that evoked drinking in rats. Although the larger lesions extended up to $3.0 \mathrm{~mm}$ from the center of the electrode and higher current levels were required to excite more distal neuronal elements, all animals continued to drink when stimulated. It was concluded that preexisting, stable characteristics of animals and not the precise neuroanatomical locus of the electrode accounted for the response to hypothalamic stimulation.

\footnotetext{
'Send reprint requests to Dr. Elliot S. Valenstein, Neuroscience Laboratory Building, 1103 E. Huron, University of Michigan, Ann Arbor, MI 48109.
} 
Species as well as individual differences have also been shown to be important in determining the responses to brain stimulation. In rats, for example, it was noted that hypothalamic stimulation evoked a strong tendency to carry both edible and inedible objects when rewarding stimulation was administered on one side of a two compartment testing chamber. In contrast, animals such as guinea pigs, which do not normally build nests and retrieve objects, did not carry objects under comparable testing conditions, although they self-stimulated by running back and forth in the test chamber [44]. Other examples of species differences in response to hypothalamic stimulation are described by Valenstein [59]. Considered together, these observations support the conclusion that many factors besides electrode placement can influence the type of behavior produced in response to hypothalamic stimulation. These factors include characteristics of individual animals within a species and differences in the natural behavior between species.

Current research in this laboratory has concentrated on factors that influence individual responses to electrical stimulation of the brain. We have consistently observed that there is no relationship between ad lib food and water consumption and the probability that brain stimulation would evoke eating or drinking. The possibility that genetic factors may contribute to individual differences has been suggested by reports that differences in self-stimulation rate are inherited in rats $[34,35]$ and mice $[14,15,16,17,18,19]$. In our earlier studies we had used Sprague-Dawley rats exclusively, but recently we have used rats from other strains. Preliminary observations seemed to indicate that the probability of evoking eating and drinking by hypothalamic stimulation was higher in Long-Evans than Sprague-Dawley rats [3]. The purpose of the present investigation was to determine if the strain differences in probability of evoking eating and drinking by hypothalamic stimulation were reliable and if so, what factors might contribute to the differences.

\section{METHOD}

\section{Subjects, Surgery, Stimulation Parameters}

The subjects were 51 male Sprague-Dawley albino rats (Holtzman Co., Madison, WI) and 49 Long-Evans hooded rats (Simonsen Co., Gilroy, CA and Charles River, Wilmington, MA). All animals weighed between $250-400 \mathrm{~g}$ at the time of surgery. They were bilaterally implanted with twisted bipolar stainless steel electrodes (Plastic Products Co., Roanoke, VA, No. MS 303/1, $0.25 \mathrm{~mm}$ dia.) bared of insulation only at the adjacent tips of the wire. Equithesin (Jensen-Salsbery Lab, Kansas City, MO) anesthetic (2.7 $\mathrm{cc} / \mathrm{kg}$ ) was used, and the electrodes were fixed to the skull by stainless steel screws and dental acrylic. With the skull held level between bregma and lambda, stereotaxic coordinates were $3.3 \mathrm{~mm}$ posterior to bregma, $1.4 \mathrm{~mm}$ to each side of the sagittal suture, and $8.3 \mathrm{~mm}$ below the dorsal surface of the skull. Animals were stimulated with $20 \mathrm{sec}$ trains of $60 \mathrm{~Hz}$ sine waves from a constant current source, alternating with $15 \mathrm{sec}$ inter-trial-intervals. The onset and duration of electrical stimulation of the lateral hypothalamus (ESLH) was always controlled by automatic, programming equipment.

\section{Procedure}

Animals were housed in individual cages with food and water always available. The vivarium was temperature regulated and lights were maintained on a 12-12 hour, dark-light cycle. One week following surgery, animals were placed in a $20.5 \times 26.5 \times 42.5 \mathrm{~cm}$ Plexiglas chamber with a cardboard floor. Food pellets (P.J. Noyes Co., Lancaster, NH, $45 \mathrm{mg}$ ) were scattered on the floor, and a filled water bottle with a metal drinking tube was attached to one wall. Following 30 min of habituation to the test chamber, all animals were screened for evoked eating and drinking. One electrode was randomly chosen for stimulation at the beginning of the screening trials. Animals were initially exposed to a $4 \mu \mathrm{A}$ current that was increased by $1 \mu \mathrm{A}$ at each subsequent trial until the animal either ate or drank, or until the stimulation seemed to agitate the rat excessively. Observations were made very carefully to assure that the animals actually ingested food or water, rather than only crumbling the food or lapping around the drinking tube.

If a rat regularly engaged in consummatory behavior during stimulation and did not eat or drink during the inter-trial intervals, it was designated positive, and the screening procedure was then repeated for the other electrode. Rats that showed no eating or drinking at either electrode were rescreened within $48 \mathrm{hr}$ using the same procedure. Animals that failed to show consummatory behavior after 2 screening sessions, and animals that exhibited disruptive motor responses that precluded consummatory behavior, were classified negative. The 6 rats (3 Long-Evans and 3 Sprague-Dawley) that seemed to exhibit some tendency to eat or drink during the initial screening, but did not continue to display these behaviors during the later threshold tests, were also classified as negative.

Positive rats were placed on a schedule of threshold testing. The threshold test consisted of a modified "staircase titration" procedure for obtaining the minimum stimulation intensity capable of evoking eating or drinking. The current intensity was raised in $1 \mu \mathrm{A}$ steps, from an initial point $3 \mu \mathrm{A}$ below the animal's screening threshold, until eating or drinking occurred. The same current intensity was then repeated on the next trial. If eating or drinking did not recur, the intensity was raised again. Following two consecutive positive trials, the current was reduced by $3 \mu \mathrm{A}$ and the process was repeated twice more. The three intensities at which an animal ate or drank twice in succession were averaged for the "daily threshold." Threshold tests at one electrode site were administered over seven consecutive sessions spaced 24-48 hrs apart and then the other electrode site was similarly tested. The 5 Long-Evans rats that initially ate or drank at only one of their two electrodes were retested at the negative electrode after the seven threshold tests of the positive electrode were completed. Two of the 5 animals reliably ate or drank during stimulation and threshold tests were then administered. Of the initial 100 animals, 6 Long-Evans and 5 Sprague-Dawley rats died before screening or threshold testing was completed and have been eliminated from the results. Therefore, behavioral data were obtained from 43 Long-Evans and 46 Sprague-Dawley rats.

\section{Histology}

Upon completion of behavioral testing, the animals were overdosed with Equithesin and perfused through the heart with saline and $10 \%$ Formalin solution. Histological sections $(60 \mu \mathrm{m})$ of the frozen brains were used to locate the electrode tips. Animals that died before their brains could be perfused and ambiguous cases produced by movement of the electrodes or tearing of tissue were not included in the histological analysis. 


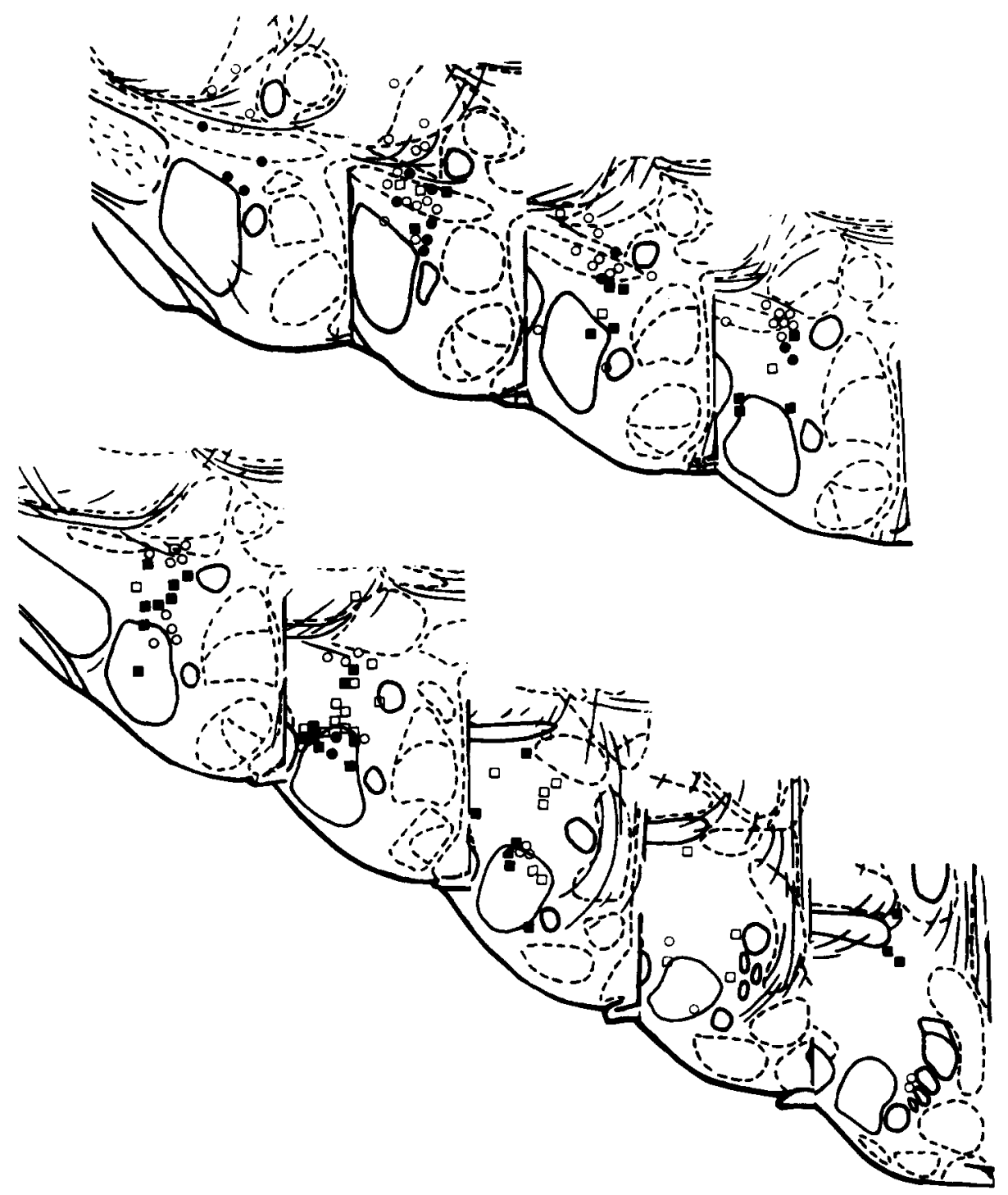

FIG. 1. The positive (evoked eating and/or drinking) and negative electrode placements: Long-Evans positive ( $(\square)$, negative ( $\square$ ); Sprague-Dawley positive $(\bullet)$, negative $(0)$ [31].

RESULTS

Confirming our preliminary observations, a much larger percentage of the Long-Evans rats ate and drank during ESLH than did the Sprague-Dawley animals. Because there was no difference, the results from the Long-Evans rats obtained from the two suppliers were combined. While $56 \%(24$ of 43) of the Long-Evans rats ate or drank during ESLH, only $22 \%$ (10 of 46 ) of the Sprague-Dawley rats exhibited these evoked behaviors. This difference between the strains is statistically significant $\left(p<0.001, \chi^{2}=10.93 ; \mathrm{df}=1\right)$. There were no differences in the animals that did exhibit evoked behaviors, however, as the majority $(74 \%)$ of positive animals in both strains ate and drank during stimulation even though no effort was made to modify responses by removing either food or water as had been done in earlier experiments [62].

No difference in the behavior of negative animals could be detected. There was no greater incidence of disruptive behaviors, such as turning or jumping, in the negative animals from either strain, nor did the two strains differ in sensitivity to electrical stimulation. A $t$-test comparing the mean threshold for evoking eating and drinking between the strains was nonsignificant (Long-Evans: mean $=7.85 \mu \mathrm{A}$, $\sigma=5.04 \mu \mathrm{A}$; Sprague-Dawley: mean $=8.34 \mu \mathrm{A}, \sigma=4.33 \mu \mathrm{A}$ ).

In addition to strain differences, the data also confirmed the importance of individual differences as demonstrated by the finding that all 10 of the Sprague-Dawley animals that ate or drank when one electrode was stimulated exhibited the same response when the second electrode was stimulated, in spite of the relatively low probability of success characteristic of this strain. Only 3 of the 24 positive Long-Evans rats failed to show eating or drinking from both electrodes.

A careful histological analysis was undertaken in order to evaluate the possibility that the strain differences might be explained by differences in electrode placement. Figure 1 presents the anatomical distribution of 137 electrode sites in 69 (34 Long-Evans; 35 Sprague-Dawley) animals of the 89 which completed the experiment. Histology was not available for 20 rats, but the ratio of positive to negative LongEvans and Sprague-Dawley animals included in the histolog- 


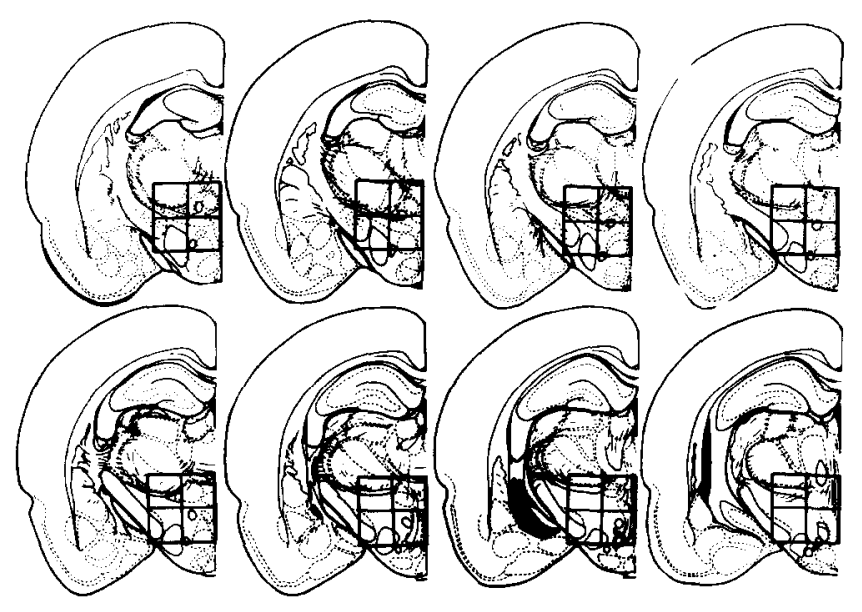

FIG. 2. The matrix of boxes used in the histological analysis. The 1.0 $\mathrm{mm}$ boxes are drawn to scale. The top 4 sections were designated anterior; the bottom sections were designated posterior. The electrode placements that were charted on the middle Plate of Fig. 1 were randomly reassigned to the adjacent Plate in either the anterior or posterior group [31].

ical analysis was almost identical to that achieved by the total group in each strain. Inspection of the histological results revealed no striking differences in electrode placements between the two strains that could account for the differences in evoked consummatory behavior. In both strains, electrode sites were distributed throughout the anteriorposterior extent of the lateral hypothalamus-medial forebrain bundle, the perifornical area, the Zona Incerta and the $\mathrm{H}_{1}$ and $\mathrm{H}_{2}$ Fields of Forel, all regions where stimulation is known to evoke eating and drinking [22].

A more detailed comparison of the electrode placements in the two strains was achieved by dividing the brain region that encompassed the electrode placements into a matrix of boxes. All but 5 electrode placements were included within this matrix. As shown in Fig. 2, a transparent template consisting of 4 boxes of equal area were placed on the brain schematics used to chart the electrode placements shown in Fig. 1. The distribution of electrode placements was further divided into an anterior and posterior set. Positive and negative electrode sites that fell within each square were recorded separately for each strain. Thus, all electrode placements were recorded in either the anterior or posterior set of 4 boxes, which were designated as dorsolateral, dorsomedial, ventrolateral, and ventromedial, respectively. It should be noted on Fig. 1 that no electrode placements were located in the most ventral hypothalamic regions and therefore the matrix was positioned so that the boxes designated as ventral did not extend below the fornix columns. Consequently, the dorsal boxes, particularly in the anterior set, extended considerably above the Zona Incerta into the ventral thalamus (Fig. 2).

A 4-way analysis of variance (ANOVA) for unweighted means was performed on the proportion of positive and negative electrode sites by strain and by the three planes (medial-lateral, dorsal-ventral, and anterior-posterior). The ANOVA indicated that only 3 factors significantly affected success rate: (1) strain $(\mathrm{F}=4.28, p<0.05)$; (2) dorsal-ventral $(\mathrm{F}=4.39, p<0.05)$; and (3) dorsal-ventral $\times$ anterior-posterior $(\mathrm{F}=4.26, p<0.05)$. The strain difference reflects the fact that the Long-Evans rats had a significantly higher percentage of success. Moreover, the strain difference did not interact significantly with any placement dimension, suggesting that the strains were not differentially affected by electrode placement. The significance of the other two factors indicates that rats of both strains had a higher proportion of negative sites in the dorsal boxes and in particular, in the antero-dorsal boxes of our matrix. The interpretation of the latter finding, however, must take into consideration the specific location of the dorsal boxes in the anterior and posterior halves of our distribution, as described above.

\section{DISCUSSION}

The present results demonstrate that there are significant strain differences in the probability of evoking eating and drinking by electrical stimulation of the lateral hypothalamus. The probability of evoking these consummatory behaviors was more than two and one-half times greater with Long-Evans than with Sprague-Dawley male rats. The reliability of these results is substantiated by noting that the success rate of $22 \%$ in the present Sprague-Dawley sample is very similar to the $25 \%$ success reported 12 years earlier [62] with animals obtained from the same supplier (Holtzman Co.). The recent report [50] that $34 \%$ (10 of 29) of the Sprague-Dawley male rats from a different supplier (Zivic Miller) exhibited evoked eating or drinking is also not significantly different (Chi Square) from our present results.

Although the present study was addressed primarily to strain differences, the results also provide strong support for earlier statements about the important contribution of individual differences $[43,58,63,67]$. Even though only $22 \%$ of the Sprague-Dawley rats ate or drank in response to hypothalamic stimulation, all 10 of the animals positive at one electrode site exhibited the same behavior when stimulated at the second electrode. The probability of this occurring by chance is less than one in a million (binomial expansion). This result cannot be attributed to similarity of the placements as in several instances the two electrodes in positive animals were even located in different boxes of the matrix.

An awareness of these previously unreported strain differences will make it possible to choose experimental subjects that provide a greater return on the effort expended in implanting electrodes and screening animals. In addition to the obvious practical advantages, such strain differences could provide important leads for investigating the mechanisms underlying eating and drinking evoked by brain stimulation.

At this time, attempts to explain the strain differences in incidence of evoked ingestive behavior must be speculative. Nevertheless, the available evidence indicates that the more obvious explanations can probably be ruled out. As already noted, for example, the strain differences in evoked behavior cannot be attributed to differences in location of the stimulating electrodes. Our histological and statistical analyses revealed that electrode placement could not account for the significant strain differences in evoked eating and drinking. Nor is there any reason to attribute the strain differences in evoked behavior to differences in normal consumption rates as animals of both strains consume the same amount of food or water in their home cages and exhibit the same rate of body weight growth.

It also seems unlikely to us that the strain differences in evoked behavior could be attributed to differences in rearing. We have already noted that essentially the same percentage of success was achieved with Sprague-Dawley rats obtained from different suppliers: In addition, there were no differences in the present results with the Long-Evans rats 
purchased from two suppliers. Furthermore, we have observed that the probability of evoking eating or drinking was not different whether the Sprague-Dawley animals were bred and raised in our laboratory or purchased from suppliers as adults. Lastly, there are several reports that neither prior experience in the testing chambers [61] nor rearing animals without solid food [64] affects the probability of successfully evoking eating by hypothalamic stimulation. Therefore, we consider it very unlikely that differences in laboratory routines could account for the strain differences reported here, but we are not contesting the reports that experimental interventions such as aversive conditioning can influence the incidence of evoked behavior [70].

Five hypotheses have been proposed to explain why hypothalamic stimulation evokes eating and drinking. For the most part, these hypotheses represent differences in focus and may actually be complementary, rather than mutually exclusive. Historically, the first hypothesis proposed was that stimulation evoked hypothalamic neural mechanisms regulating specific biological drives. According to this view, animals eat or drink because the stimulation makes them hungry or thirsty $[28,41]$. Many arguments are advanced in support of this hypothesis, but generally considered most persuasive is the claim that an instrumental, food-rewarded response, learned when an animal is food deprived, is readily used by the same animals when receiving hypothalamic stimulation [21]. Also considered compelling by some is the demonstration that several drugs and hormones that increase or decrease food or water consumption have the same effect on eating and drinking evoked by hypothalamic stimulation [23].

The hypothesis that electrical stimulation activates the specific neural substrate of hunger and thirst has been seriously challenged by demonstrations that: (1) stimulation from a given electrode typically evokes several different behaviors [62,63]; (2) animals eating or drinking in response to hypothalamic stimulation behave very differently than hungry or thirsty animals $[51,58] ;(3)$ the anatomical distribution of electrode placements capable of evoking eating or drinking is much more widespread than originally postulated and does not correspond with the more lateral hypothalamic "feeding and drinking area"' defined by lesion studies [42,55] and (4) non-specific activating stimulation, such as tailpinch, also evokes eating and other behaviors ([2], see also [49] for a critical review). This is not the place to attempt to resolve the controversy, but in the present context, it is important to appreciate that even if hypothalamic stimulation does activate hunger and thirst, this would not explain why animals that normally consume equal amounts of food and water should differ so strikingly in the probability of displaying eating or drinking in response to such stimulation.

It has also been hypothesized that hypothalamic stimulation activates responses that are linked to the neural mechanisms of reinforcement or reward [27]. According to such "response-oriented theories" of evoked behavior, hypothalamic stimulation evokes eating, drinking and other behaviors by activating brainstem motor mechanisms for these responses while simultaneously bringing into play reinforcement mechanisms that have evolved to maintain biologically significant behaviors and strengthen the probability of their recurring under similar conditions $[12,27]$. This view is supported by the demonstration that eating, drinking, aggression, grooming and other behaviors evoked by hypothalamic stimulation can also be evoked by stimulation of sites in the brainstem and cerebellum $[4,10,11,32,48,65,72]$. It has also been claimed that, like the behaviors seen during hypothalamic stimulation, some of the behaviors evoked by brainstem stimulation are "goal directed" and therefore "motivated," but the data are sparse and more work is admittedly required [12]. The fact that lesions posterior to hypothalamic electrode placements may block the behavior evoked by stimulation is considered additional evidence of the importance of the more caudal brainstem sites $[9,13,63]$. One problem is that most of these demonstrations do not rule out the importance of ascending connections, but in some instances, caudally directed axons have been traced from hypothalamic electrodes to brainstem sites where stimulation proved to be capable of evoking the same behavior evoked by hypothalamic stimulation $[5,20]$.

Clearly there is considerable evidence that the substrate of many of the behaviors evoked from hypothalamic sites are represented at different levels of the neuraxis, but at present it is not possible to relate the strain differences reported here to this body of data. There is no evidence of strain (or individual) differences either in the brainstem substrate of eating and drinking or in the relative strength of hypothalamicbrainstem connections. Some of this information would be difficult to obtain in a quantitative form, but it would be possible at least to determine if the probability of evoking particular behaviors by stimulation of hypothalamic and brainstem sites in the same animal is correlated. It would, however, require additional experimentation to interpret such a relationship if it were found to exist.

The behaviors evoked by stimulation have also been explained by referring to hypothalamic mechanisms thought to modulate sensory and motor responsiveness. In an extensive series of studies, Flynn and his co-workers have provided evidence that hypothalamic stimulation in cats can induce sensory-motor changes which are believed to increase the capacity of stimuli to evoke particular responses $[6,25,36]$. Similarly, Smith [52] observed that stimulation, which evokes such responses as eating, gnawing, drinking, or attack behavior in rats, increases sensitivity of the perioral region. The phenomenon of unilateral "sensory neglect" produced by unilateral hypothalamic lesions has also provided evidence of mechanisms regulating responsiveness to stimuli $[33,37,38,39,40,54,56]$.

Implicit in the stimulation and lesion studies cited above is the assumption that there exist hypothalamic mechanisms for modulating the incentive value of stimuli associated with the satisfaction of specific motivational states. These mechanisms are not thought to exert their influence directly on sensory or motor systems, but rather to facilitate sensorymotor integration $[29,56]$. The view that hypothalamic stimulation only increases responsiveness to relevant stimuli, however, has been challenged by evidence that stimulation produces a nonspecific increase in reactivity even to neutral stimuli $[1,8]$. This issue is not yet resolved, but at present there is no available evidence of strain differences in the capacity of hypothalamic stimulation to produce sensorymotor changes that can explain the strain differences reported here. It might be useful to note, however, that only those rats which display evoked eating, drinking, gnawing or attack are reported to exhibit increased perioral sensitivity during hypothalamic stimulation [52], but it is not clear whether these results reflect electrode placement or animal differences.

Another explanatory hypothesis of evoked behaviors has emerged from evidence that the dopamine pathways, which course through the hypothalamus [24,57], are important for 
both self-stimulation and stimulation-evoked behaviors. Destruction, or pharmacological blocking, of the ascending dopamine pathways have been shown to impair selfstimulation (see [69] for review) and the capacity of hypothalamic stimulation to evoke eating $[45,46,47,50]$. It is still not clear, however, whether the demonstrations that evoked eating is impaired after interference with dopamine activity can logically be used to argue that the initiation of such behavior is also controlled by dopamine activity. One problem here is that destruction of dopamine pathways has been shown to produce numerous non-specific effects on motor activity. To control for some of the non-specific effects, Paul Fray in this laboratory has been studying the effect of unilateral destruction of dopamine pathways on self-stimulation and evoked behavior obtained from bilateral electrodes.

As yet, there is little evidence that individual or strain differences in evoked eating and drinking can be attributed to differences in dopamine mechanisms. A recent report, however, claiming evidence that bilateral differences in selfstimulation rate are correlated with asymmetry in dopamine activity provides an interesting lead in this regard [26]. Although this finding has not yet been duplicated for evoked eating and drinking, it remains a possibility that some aspect of dopamine activity may underlie individual and strain differences in propensity to exhibit such behaviors. Pursuing this possibility, Susan Bachus in this laboratory, has preliminary observations suggesting a relationship between the type of stereotyped behaviors displayed following administration of the catecholamine mimetic, amphetamine, and the probability that eating and drinking will be evoked by hypothalamic stimulation.

Lastly, a relationship between emotionality and the probability of evoking eating and drinking by hypothalamic stimulation has been suggested $[53,71]$. Soper and Wise [53] have reported that animals, which do not normally exhibit evoked eating, will eat in response to hypothalamic stimulation while under the influence of the tranquilizer, diazepam. The conclusion that the tranquilizer counteracted the influence of "emotional systems involved in suppression of behavior" has been challenged, however, by the subsequent report that diazepam does not similarly facilitate evoked drinking and therefore the facilitation of evoked eating might be caused by the recognized effects of this drug on appetite, rather than its effect on emotionality [66].

Although interpretation of the diazepam effect on evoked behavior is unclear, we believe that some aspect of emotionality may be correlated with the strain differences re- ported here. We have observed that Long-Evans rats are more resistant to handling and vocalize more frequently than Sprague-Dawley rats. Also, it has been reported that LongEvans rats locomote less than Sprague-Dawley rats in an open field test, a result which also reflects the greater emotionality of the Long-Evans animals [7]. In an unpublished study of strain differences in tail-pinch evoked eating, Mark Litchman, working in this laboratory, observed that LongEvans male rats appeared to respond more emotionally when the paper clip was placed on their tails than did the Sprague-Dawley male rats. The emotionality of the LongEvans rats was reflected in the significantly greater amount of urination and fecal boli, the looser consistency of the fecal boli, and in the greater amount of time orienting to the paper clip rather than to food. Possibly because of their high levels of emotionality, the Long-Evans rats spent significantly less time eating and a significantly lower percentage of animals ate in response to tail pinch than did the Sprague-Dawley animals. In a collaborative study with Israel Lieblich and Edna Cohen of Hebrew University, one of the present authors (E. S. V.) has found a significant difference between two inbred strains of rats in the probability of evoking eating and drinking by hypothalamic stimulation. Preliminary results also showed that animals from the strain with the greater amount of eating and drinking evoked by the brain stimulation exhibited less eating in response to tail pinch.

We are faced with the problem of understanding why rats, which exhibit significantly more eating evoked by hypothalamic stimulation, should eat less during tail-pinch. Although we tried to resist the temptation of employing the much overly used inverted $U$ explanation, the opposite direction of the strain differences in eating evoked by hypothalamic stimulation and tail pinch appears to conform to this model. It is not unreasonable to assume that an optimal level of activation facilitates eating while very high levels interfere with this behavior. Conceivably, the Long-Evans rats, because they are more active normally, exhibit a higher probability of eating with the relatively mild hypothalamic stimulation and a lower probability during the more intense tail-pinch stimulation while the Sprague-Dawley animals exhibit the reverse pattern.

\section{ACKNOWLEDGEMENT}

We are pleased to acknowledge the statistical assistance of Dr. Norman Henderson and Mr. Buda Martonyi's assistance with the programming equipment and histology.

\section{REFERENCES}

1. Ackil, J. E., M. J. Levinson and G. P. Frommer. Hypothalamic influences on sensory reinforcement. Physiol. Behav. 14: 133$142,1975$.

2. Antelman, S. M., N. E. Rowland and A. E. Fisher. Stimulation-bound ingestive behavior: $A$ view from the tail. Physiol. Behav. 17: 743-748, 1976.

3. Bachus, S. E. and E. S. Valenstein. Individual behavioral responses to hypothalamic stimulation persist despite destruction of tissue surrounding electrode tip. Physiol. Behav. 23: 421-426, 1979.

4. Ball, G. G., D. J. Micco and G. G. Berntson. Cerebellar stimulation-bound oral behaviors and self-stimulation. Physiol. Behav. 13: 123-127, 1974.

5. Bandler, R. J., C. C. Chi and J. P. Flynn. Biting attack elicited by stimulation of the ventral midbrain tegmentum of cats. Science 177: 364-366, 1972.
6. Bandler, R. and J. P. Flynn. Visual patterned reflex present during hypothalamically elicited attack. Science 171: 817-818, 1971.

7. Barrett, R. J. and O. S. Ray. Behavior in the open field, Lashley III maze, shuttle-box, and Sidman avoidance as a function of strain, sex, and age. Devl Psychol. 3: 73-77, 1970.

8. Beagley, W. K. and T. L. Holley. Hypothalamic stimulation facilitates contralateral visual control of a learned response. Science 196: 321-322, 1977.

9. Berntson, G. G. Blockade and release of hypothalamically and naturally elicited aggressive behaviors in cats following midbrain lesions. J. comp. physiol. Psychol. 81: 541-554, 1972.

10. Berntson, G. G. Attack, grooming, and threat elicited by stimulation of the pontine tegmentum in cats. Physiol. Behav. 11: 81-87, 1973.

11. Berntson, G. G. and H. C. Hughes. Medullary mechanisms for eating and grooming behaviors in the cat. Expl Neurol. 44: 255256, 1974. 
12. Berntson, G. G. and D. J. Micco. Organization of brain stem behavioral systems. Brain Res. Bull. 1: 441-482, 1976.

13. Bergquist, E. H. Output pathways of hypothalamic mechanisms for sexual, aggressive, and other motivated behaviors in opossum. J. comp. physiol. Psychol. 70: 389-398, 1970.

14. Cazala, P. Extinction du comportement d'autostimulation chez trois lignées de souris consanguines: Influence du délai séparant la période de renforcement de la séance de l'extinction. Physiol. Behav. 21: 701-704, 1978.

15. Cazala, P., Y. Cazals and B. Cardo. Hypothalamic selfstimulation in three inbred strains of mice. Brain Res. 81: 159 167, 1974.

16. Cazala, P. and J. - L. Guenet. The role of genetic factors in the determination of self-stimulation behaviour in the mouse: Backcross analysis. Behav. Proc. 1: 93-97, 1976.

17. Cazala, P. and J. -L. Guenet. Participation of the dilute locus in the genetic control of self-stimulation behaviour in the mouse. Neurosci. Lett. 6: 361-364, 1977.

18. Cazala, P. and J. -L. Guenet. Effects of the albino gene on self-stimulation behavior in the lateral hypothalamus in the mouse. Physiol. Behav, 22: 7-9, 1979.

19. Cazala, P. and J. -L. Guenet. The recombinant inbred strains: A tool for the genetic analysis of differences observed in the selfstimulation behaviour of the mouse. Physiol. Behav. 24: 1057$1060,1980$.

20. Chi, C. C. and J. P. Flynn. Neural pathways associated with hypothalamically elicited attack behavior in cats. Science 171: 703-705, 1971.

21. Coons, E. E., M. Levak and N. E. Miller. Lateral hypothalamus: Learning of food-seeking responses by electrical stimulation. Science 150: 1320-1321, 1965.

22. Cox, V. C. and E. S. Valenstein. Distribution of hypothalamic sites yielding stimulus-bound behavior. Brain Behav. Evolut. 2: 359-376, 1969.

23. Devor, M. G., R. A. Wise, N. W. Milgram and B. G. Hoebel. Physiological control of hypothalamically elicited feeding and drinking. J. comp. physiol. Psychol. 73: 226-233, 1970.

24. Fallon, J. H. and R. Y. Moore. Catecholamine innervation of the basal forebrain: IV. Topography of the dopamine projection to the basal forebrain and neostriatum. J. comp. Neurol. 180: 545-580, 1978.

25. Flynn, J. P., S. B. Edwards and R. J. Bandler. Changes in sensory and motor systems during centrally elicited attack. Behav. Sci. 16: 1-19, 1971.

26. Glick, S. S., L. M. Weaver and R. C. Meibach. Lateralization of reward in rats: Differences in reinforcing thresholds. Science 207: 1093-1094, 1980.

27. Glickman, S. E. and B. B. Schiff. A biological theory of reinforcement. Psychol. Rev. 74: 81-109, 1967.

28. Hess, W. R. The Functional Organization of the Diencephalon. New York: Grune \& Stratton, 1957.

29. Hoyman, L., G. D. Weese and G. P. Frommer. Tactile discrimination performance deficits following neglect-producing unilateral lateral hypothalamic lesions in the rat. Physiol. Behav. 22: 139-147, 1979.

30. Karli, P., M. Vergnes and F. Didiergeorges. Rat-mouse interspecific aggressive behavior and its manipulation by brain ablation and by brain stimulation. In: Biology of Aggressive Behavior, edited by S. Garattini and E. B. Siggs. Amsterdam: Excerpta Medica Foundation, 1969, pp. 47-55.

31. König, J. F. R. and R. A. Klippel. The Rat Brain: A Stereotaxic Atlas of the Forebrain and Lower Parts of the Brain Stem. Huntington, N.Y.: Robert E. Krieger, 1970.

32. Kooy, D. van der and A. G. Phillips. Trigeminal substrates of intracranial self-stimulation. Science 196: 447-449, 1977.

33. Levitt, D. R. and P. Teitelbaum. Somnolence, akinesia and sensory activation of motivated behavior in the lateral hypothalamic syndrome. Proc. natn. Acad. Sci. U.S.A. 72: 2819-2823, 1975.

34. Lieblich, I., E. Cohen and A. Beiles. Selection for high and for low rates of self-stimulation in rats. Physiol. Behav. 21: 843849, 1978.
35. Lipp, H. P. Differential hypothalamic self-stimulation behavior in Roman High-Avoidance and Low-Avoidance rats. Brain Res. Bull. 4: 553-559, 1979.

36. MacDonnell, M. and J. P. Flynn. Control of sensory fields by stimulation of hypothalamus. Science 152: 1406-1408, 1966.

37. Marshall, J. F., D. Levitan and E. M. Stricker. Activationinduced restoration of sensorimotor functions in rats with dopamine depleting brain lesions. J. comp. physiol. Psychol. 90: 536-546, 1976.

38. Marshall, J. F., J. S. Richardson and P. Teitelbaum. Nigrostriatal bundle damage and the lateral hypothalamic syndrome. $J$. comp. physiol. Psychol. 87: 808-830, 1974.

39. Marshall, J. F. and P. Teitelbaum. Further analysis of sensory inattention following lateral hypothalamic damage in rats. $J$. comp. physiol. Psychol. 86: 375-395, 1974.

40. Marshall, J. F. and P. Teitelbaum. New considerations in the neuropsychology of motivated behaviors. In: Handbook of Psy. chopharmacology, Vol. 7: Principles of Behavioral Pharmacology, edited by L. L. Iversen, S. D. Iversen and S. H. Snyder. New York: Plenum, 1977, pp. 201-229.

41. Miller, N. E. Commentary. In: Brain Stimulation and Motivation, edited by E. S. Valenstein. Glenview, IL: Scott, Foresman \& Co., 1973, pp. 53-68.

42. Mufson, E. J. Pathways disrupted in aphagia and adipsia following diencephalic damage. Brain Behav. Evolut. 17: 310-338, 1980.

43. Panksepp, J. Aggression elicited by electrical stimulation of the hypothalamus in albino rats. Physiol. Behav. 6: 321-329, 1971.

44. Phillips, A. G., V. C. Cox, J. W. Kakolewski and E. S. Valenstein. Object-carrying by rats: An approach to the behavior produced by stimulation. Science 166: 903-905, 1969.

45. Phillips, A. G. and H. C. Fibiger. Deficits in stimulationinduced feeding after intraventricular administration of 6-hydroxydopamine in rats. Behav. Biol. 9: 749-754, 1973.

46. Phillips, A. G. and H. C. Fibiger. The multivariate nature of motivated behavior elicited by electrical stimulation of the lateral hypothalamus. In: Current Studies of Hypothalamic Function, Vol. 2, edited by W. L. Veale and K. Ledaris. Basel: Karger, 1978, pp. 195-205.

47. Phillips, A. G. and R. S. Nikaido. Disruption of brain stimulation-induced feeding by dopamine receptor blockade. Nature 258: 750-751, 1975.

48. Reis, D. J., N. Doba and M. A. Nathan. Predatory attack, grooming and consummatory behaviors evoked by electrical stimulation of cat cerebellar nuclei. Science 82: 845-847, 1973.

49. Robbins, T. W. and P. J. Fray. Stress-induced eating: Fact, fiction or misunderstanding? Appetite 1: 103-133, 1980.

50. Rowland, N., D. M. Marques and A. E. Fisher. Comparison of the effects of brain dopamine-depleting lesions upon oral behaviors elicited by tail pinch and electrical brain stimulation. Physiol. Behav. 24: 273-281, 1980.

51. Schallert, T. Reactivity to food odors during hypothalamic stimulation in rats not experienced with stimulation-induced eating. Physiol. Behav. 18: 1061-1066, 1977.

52. Smith, D. A. Increase perioral responsiveness: A possible explanation for the switching of behavior observed during lateral hypothalamic stimulation. Physiol. Behav. 8: 617-621, 1972.

53. Soper, W. Y. and R. A. Wise. Hypothalamically induced eating: eating from 'non-eaters' with diazepam. T.I.T.J. Life Sci. 1: $79-84,1971$.

54. Stricker, E. M. and M. J. Zigmond. Recovery of function after damage to central catecholamine-containing neurons: A neurochemical model for the lateral hypothalamic syndrome. In: Progress in Psychobiology and Physiological Psychology, Vol. 6, edited by J. M. Sprague and A. N. Epstein. New York: Academic Press, 1976, pp. 121-188.

55. Teitelbaum, P. and A. N. Epstein. The lateral hypothalamic syndrome: recovery of feeding and drinking after lateral hypothalamic lesions. Psychol. Rev. 69: 74-90, 1962.

56. Turner, B. H. Sensorimotor syndrome produced by lesions of the amygdala and lateral hypothalamus. J. comp. physiol. Psychol. 82: 37-47, 1973. 
57. Ungerstedt, U. On the Anatomy, Pharmacology and Function of the Nigrostriatal Dopamine System. Stockholm: Kungl, Boktryckeriet P.A. Norstodt \& Soner, 1971.

58. Valenstein, E. S. Behavior elicited by hypothalamic stimulation: A prepotency hypothesis. Brain Behav. Evolut. 2: 295-316, 1969.

59. Valenstein, E. S. Stability and plasticity of motivation systems. In: The Neurosciences: Second Study Program, edited by F. O. Schmitt. New York: The Rockefeller University Press, 1970, pp. 207-217.

60. Valenstein, E. S. Brain stimulation and behavior control. In: Nebraska Symposium on Motivation, edited by J. K. Cole and T. B. Sonderegger. Lincoln: Univ. of Nebraska Press, 1975, pp. 251-292.

61. Valenstein, E. S. and V. C. Cox. Influence of hunger, thirst and previous experience in the test chamber on stimulus-bound eating and drinking. J. comp. physiol. Psychol. 70: 189-199, 1970.

62. Valenstein, E. S., V. C. Cox and J. W. Kakolewski. Modification of motivated behavior elicited by electrical stimulation of the hypothalamus. Science 159: 1119-1121, 1968.

63. Valenstein, E. S., V. C. Cox and J. W. Kakolewski. A Reexamination of the role of the hypothalamus in motivation. Psychol. Rev. 77: 16-31, 1970.
64. Valenstein, E. S. and A. G. Phillips. Stimulus-bound eating and deprivation from prior contact with food pellets. Physiol. Behav. 5: 279-282, 1970.

65. Waldbillig, R. J. Attack, eating, drinking, and gnawing elicited by electrical stimulation of rat mesencephalon and pons. $J$. comp. physiol. Psychol. 89: 200-212, 1975.

66. Watson, P. J., M. A. Short, G. L. Huenink and D. F. Hartman. Diazepam effects on hypothalamically elicited drinking and eating. Physiol. Behav. 24: 39-44, 1980.

67. Wise, R. A. Individual differences in effects of hypothalamic stimulation: The role of stimulation locus. Physiol. Behav. 6: 569-572, 1971.

68. Wise, R. A. Lateral hypothalamic electrical stimulation: Does it make animals 'hungry?' Brain Res. 67: 187-209, 1974.

69. Wise, R. A. The dopamine synapse and the notion of 'pleasure centers' in the brain. Trends in Neurosci. 3: 91-95, 1980.

70. Wise, R. A. and J. Albin. Stimulation-induced eating disrupted by a conditioned taste aversion. Behav. Biol. 9: 289-297, 1973.

71. Wise, R. A. and E. Erdmann. Emotionality, hunger, and normal eating: Implications for interpretation of electrically induced behavior. Behav. Biol. 8: 519-531, 1973.

72. Wyrwicka, W. and R. W. Doty. Feeding induced in cats by electrical stimulation of the brain stem. Expl Brain Res. 1: 152$160,1966$. 